\title{
Casas de Don Pedro (Belmez, Córdoba): menhires y Cronología
}

\author{
Casas de Don Pedro (Belmez, Córdoba): menhirs and chronology
}

\section{BEATRIZ GAVILÁN CEBALLOS ${ }^{1}$ [D, MARTÍ MAS CORNELLÀ ${ }^{2}$}

(1) Dpto. Historia, Geografía y Antropología. Universidad de Huelva. beatriz.gavilan@dhis1.uhu.es

(2) Dpto. Prehistoria y Arqueología. UNED.mmas@geo.uned.es

\section{Resumen:}

Ofrecemos los resultados de una datación sobre una muestra de carbón procedente de un hogar asociado a un monumento megalítico no funerario, concretamente dos menhires que, posteriormente, formaron parte de la cabecera del Dolmen de las Casas de Don Pedro. La fecha obtenida permite defender que los menhires debieron erigirse a comienzos del IV milenio a.C. y que el simbolismo que encarnan estaba en un punto álgido.

Palabras clave: menhires, hogares, cronología, Neolítico, IV milenio a.C., simbolismo.

\section{Abstract:}

We offer the results of a dating on a charcoal sample from a fire associated with a non-funerary megalithic monument, specifically two menhirs that later formed part of the head of the Dolmen de las Casas de Don Pedro. The date obtained allows us to defend that the menhirs must have been erected at the beginning of the 4th millennium BC and that the symbolism they embody was at a peak.

Key words: menhirs, fires, chronology, Neolithic, IV millennium BC, symbolism. 
El objetivo de este trabajo es dar a conocer una datación radiocarbónica procedente de uno de los hogares asociados a dos menhires erigidos previamente a la construcción del Dolmen de las Casas de Don Pedro. Este sepulcro megalítico se encuentra en el Alto Valle del Guadiato, en el sector NO de la provincia de Córdoba. Se localiza en las coordenadas $38^{\circ} 14^{\prime} 57,42^{\prime \prime} \mathrm{N}$ y 5 11 ' $58,35^{\prime}$ " $\mathrm{O}$, a $479 \mathrm{~m}$ snm y a unos $215 \mathrm{~m}$ de distancia del cauce del Arroyo Fresnedoso (fig. 1) poco antes de su confluencia con el Guadiato, sobre el que se eleva el conjunto calizo de Sierra Palacios, donde se encuentran dos poblados, Sierra Palacios I y Sierra Palacios II, a cuya necrópolis dolménica pertenece esta sepultura megalítica (Gavilán 2003-2004).

\section{LA EXCAVACIÓN DEL YACIMIENTO}

En el yacimiento se realizaron dos campañas de excavación arqueológica de urgencia (Gavilán 1987; Gavilán y Vera 2005), excavándose en la primera la cámara y una zanja al exterior, en la zona NO, que reveló una ocupación previa a la construcción del dolmen. En la segunda se intervino en el corredor, abriéndose también una amplia cuadrícula de $105 \mathrm{~m}^{2}$ que abarcaba todo el espacio funerario constituido por la cámara y el corredor.
Al exterior se detectó la siguiente sucesión estratigráfica de techo a base:

1. Tierra beige, arcillosa, que formaba el túmulo hasta una profundidad media de unos $-218 \mathrm{~cm}$. Sólo aparecieron algunos fragmentos de cerámica y una pieza de metal.

2. Arenas amarillas, estériles, con una potencia de unos $30 \mathrm{~cm}$.

3. Tierra anaranjada y granulosa; las zanjas de inserción de los ortostatos del dolmen cortaban esta tierra, que se depositó con anterioridad a la construcción de la cámara.

La excavación de este último nivel reveló la existencia de una fase previa a la edificación de la sepultura megalítica, que se puso de manifiesto a través de varias estructuras que se relacionan con dos menhires que, con posterioridad -en la segunda fase constructiva de la arquitectura megalítica y primera de edificación del dolmen-, pasaron a formar parte de la cabecera de la cámara megalítica. Así, la primera fase de construcción, en la que nos vamos a centrar, no da comienzo como una sepultura, sino como un monumento megalítico no funerario. Para una mayor información acerca de las distintas fases constructivas de esta arquitectura megalítica remitimos al lector a otras publicaciones (Gavilán 1987; Gavilán y Vera 2005; Barrios et al. 2014; Gavilán et al. 2020).

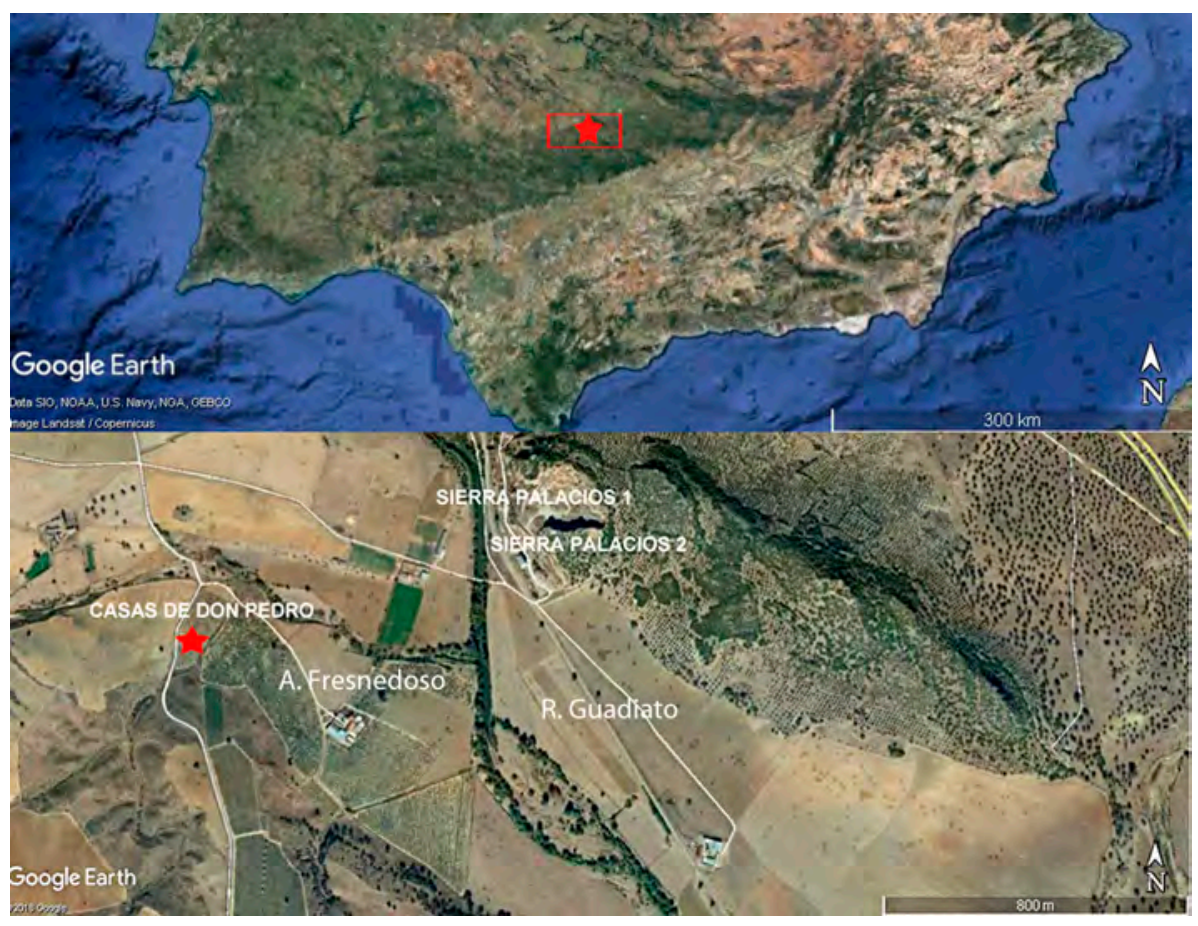

Fig. 1: Situación de Casas de Don Pedro y los poblados de Sierra Palacios. 


\section{PRIMERA FASE O FASE NO FUNERARIA}

Se inaugura, como hemos indicado, con la erección de dos menhires (fig. 2), cuyas zanjas de inserción se rellenaron de la tierra local, impidiendo esto determinar su forma y tamaño. En relación con ellos se disponían unas estructuras que reafirman su carácter como espacio simbólico y ritual: varios hoyos, un pavimento rojo, dos fosas y tres hogares, que se distribuían por los sectores $\mathrm{S}$ y $\mathrm{O}$, afectando también al $\mathrm{N}$ en la zona de contacto con el lado O (fig. 3); nada se registró en el $\mathrm{E}$, pese a que se excavó una extensión similar.

Las estructuras más antiguas corresponden a varios hoyos de 10 a $15 \mathrm{~cm}$ de diámetro y $8-10 \mathrm{~cm}$ de profundidad, que, excavados en el ángulo NO, casi circundaban el menhir más estrecho (figs. 2 y $3, n^{\circ} 2$ ), quizá el primero en alzarse. Estructuras similares quizá se dispusieron también en el ángulo SE hasta rodear el menhir, sin embargo, debieron quedar arrasadas cuando, en la segunda fase constructiva, los menhires formaron parte de la cámara funeraria (fig. 3). Todos estaban vacíos.

La siguiente estructura es un pavimento o suelo rojo que sellaba los hoyos. Se hizo a base de hematites triturada mezclada con tierra arcillosa; presentaba un espesor de entre 4-6 cm, 1,70 $\mathrm{m}$ de anchura y unos $2 \mathrm{~m}$ de longitud. Partía principalmente del menhir $\mathrm{n}^{\mathrm{o}} 2$ y no aportó resto alguno.

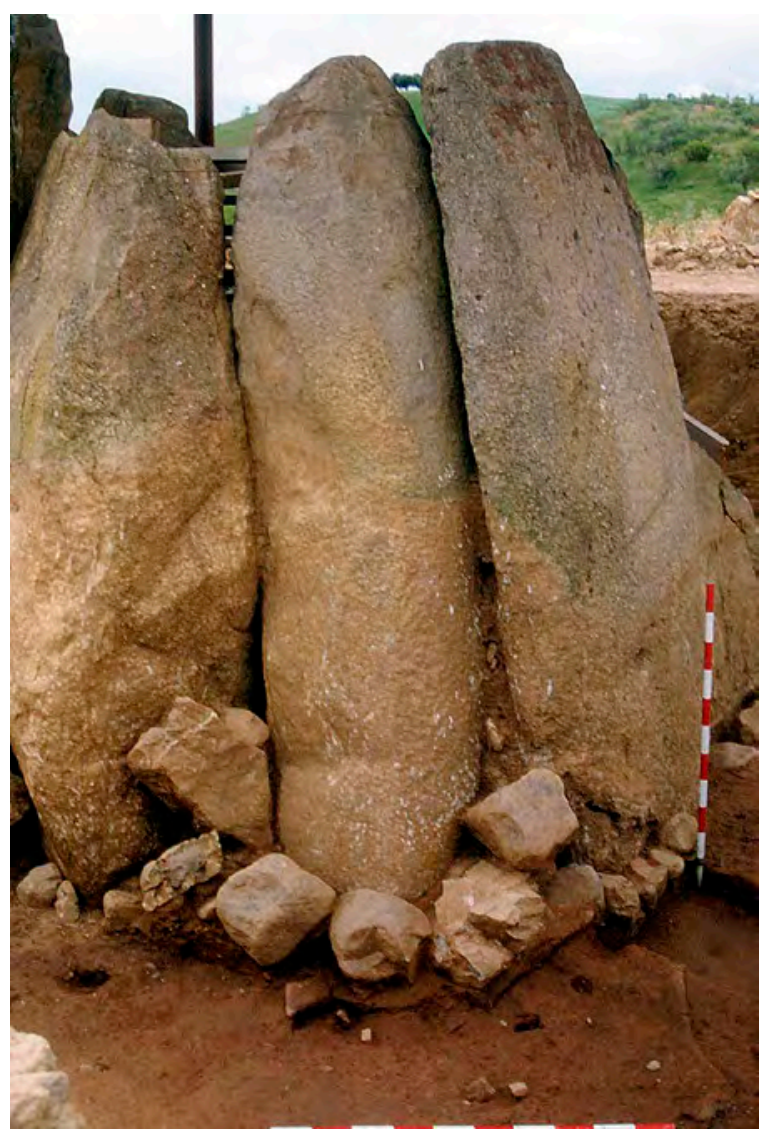

Fig. 2: Menhires de Casas de Don Pedro.
Fig. 3: Zona excavada en 2001. Planta del Dolmen de Casas de Don Pedro. Situación de los menhires y las estructuras detectadas: hoyos, pavimento rojo, fosas y hogares. Detalle del hogar del que se extrajo la muestra de carbón.

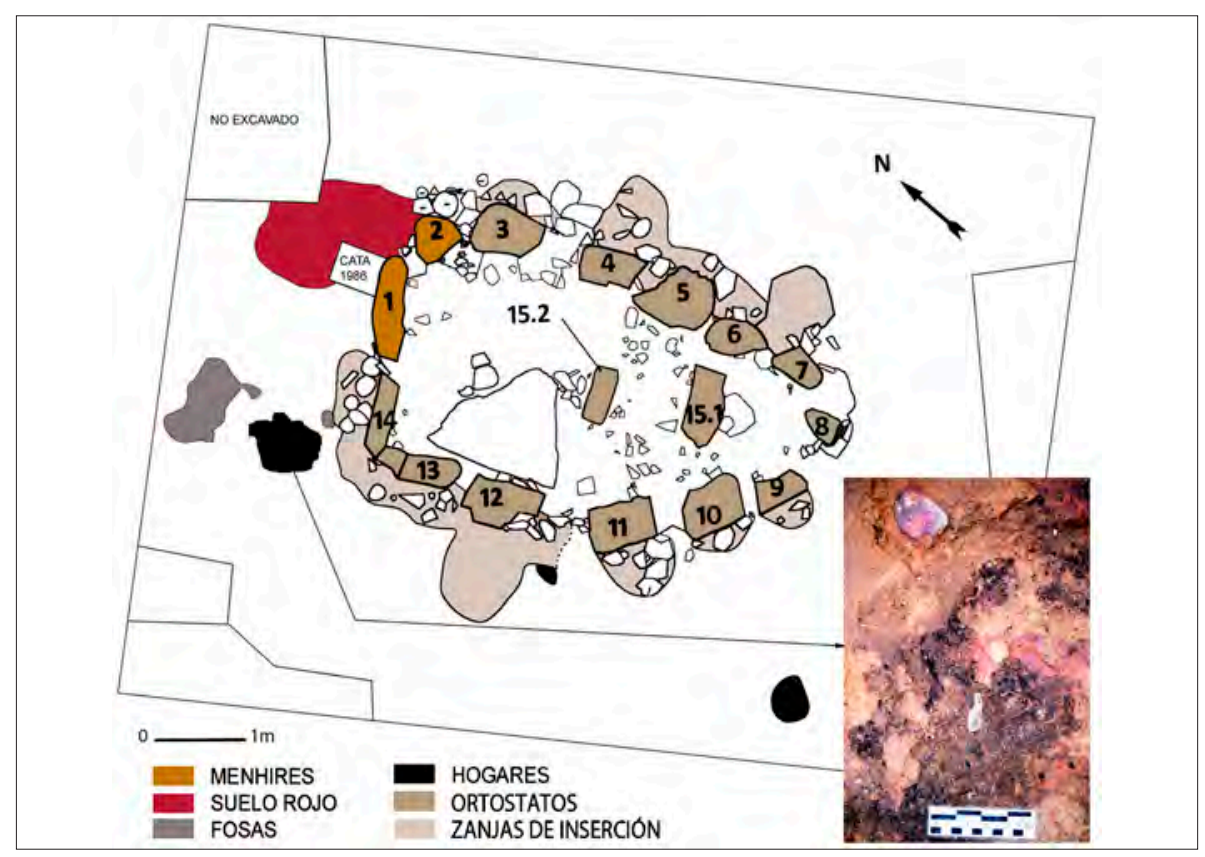




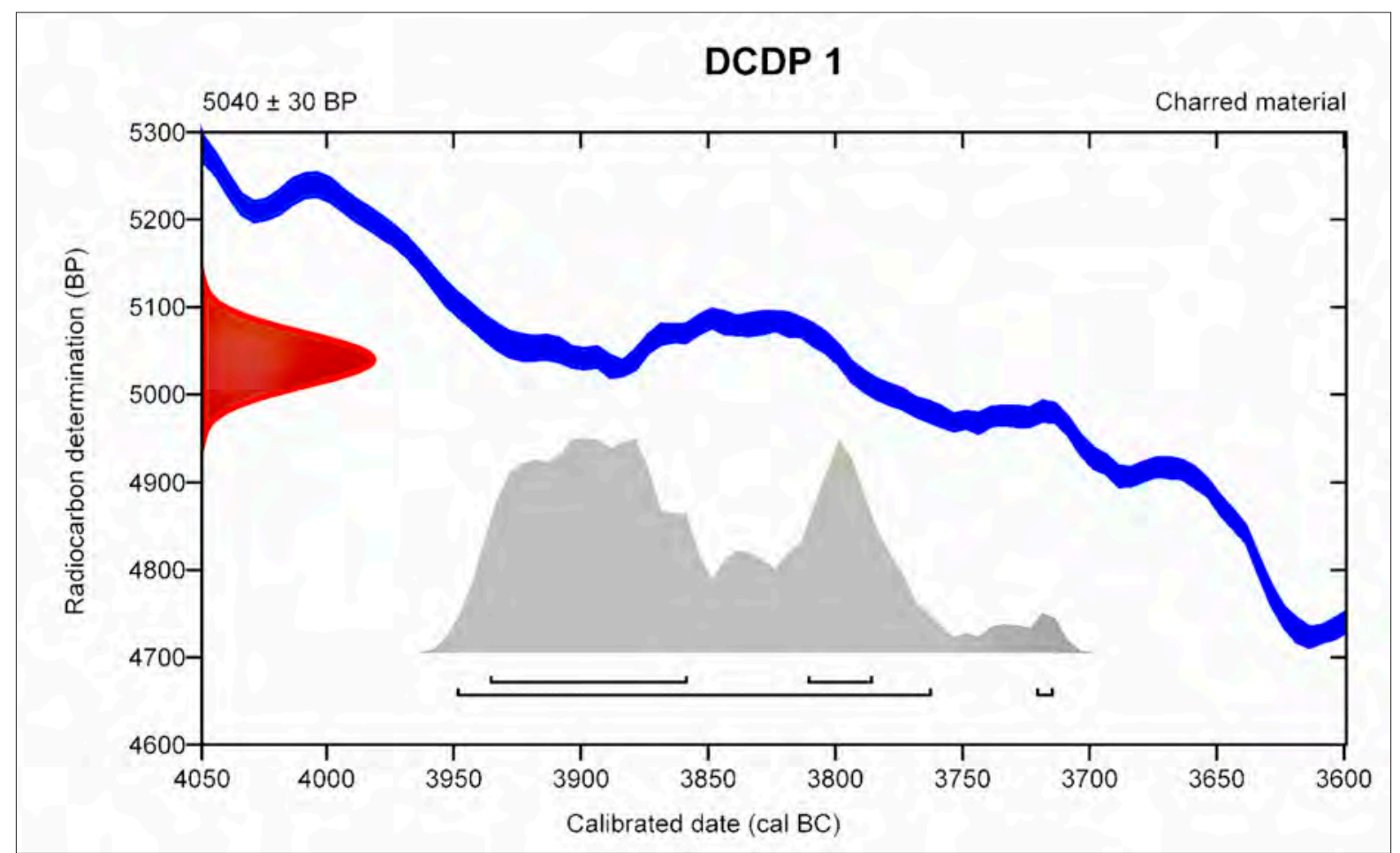

\begin{tabular}{|c|c|c|c|c|c|c|c|}
\hline Yacimiento & Sigla Lab. & Muestra & Fase & Fecha BP & Fecha BC cal 1 o & Fecha BC cal 2 o & Curva de calibración \\
\hline $\begin{array}{c}\text { Casas de Don } \\
\text { Pedro }\end{array}$ & Beta-471735 & Carbón & $1^{\text {a }}$ & $5040 \pm 30$ & $3723-3716$ & $3951-3764$ & INTCAL 13 \\
\hline
\end{tabular}

Fig. 4: Datación radiocarbónica Beta-471735.

En el sector O se detectaron dos fosas. La de mayores dimensiones proporcionó, como únicos restos, industria lítica, ósea, cerámica y varios trozos de hematites u óxido de hierro, éstos aparecieron acumulados en un saliente de la fosa.

Por último, se localizaron tres hogares que presentaban una mezcla de carbones y cinabrio (Gavilán et al. 2020); los tres se encontraban en línea recta, equidistantes unos 3,60 $\mathrm{m}$ y sin acondicionamiento previo.

Aparte de los restos recuperados en la fosa citada, aparecieron, dispersos, varios fragmentos de cerámica, industria ósea y lítica tallada, todo ello en el sector $\mathrm{O}$. Para esta primera fase no funeraria propusimos una cronología de mediados-finales del V milenio (Gavilán 2003-2004; Gavilán y Vera 2005), propuesta que no se corresponde exactamente con la datación radiocarbónica obtenida.

\section{LA DATACIÓN}

Del hogar de mayores proporciones (fig. 3) se tomó una muestra de carbón no identificado que fue introducida en doble bolsa de plástico, con etiquetado exterior en la segunda. Fue enviada en el 2017 a Beta Analytic Inc., empleándose la técnica AMS (fig. 4).

\section{CONSIDERACIONES}

Somos conscientes de que la datación presenta limitaciones puesto que se ha obtenido a partir de carbón no identificado, no procede de la fosa de cimentación de alguno de los dos menhires y no es posible defender una total sincronía entre el momento de erección de los bloques y los hogares, aunque la relación entre las estructuras y 
los menhires resulta evidente. Como también lo es que los acondicionamientos más antiguos asociados a aquellos son los primeros hoyos, que debieron excavarse tras abrir las fosas de cimentación destinadas a instalar los menhires, para, posteriormente, excavar los segundos. En un primer momento los interpretamos como agujeros para poste destinados a sustentar una estructura realizada en material perecedero (Gavilán y Vera 2005), sin embargo, su profundidad es escasa para tal finalidad y, actualmente, a la luz de lo documentado en el Dolmen de Dombate (Lestón 2011), pensamos que tal vez acogieron cantos de río, a modo de betilos, que debían reforzar el simbolismo de los menhires, sobre todo del primero en alzarse (fig. 3, $\mathrm{n}^{\mathrm{o}} 2$ ), en torno al cual se disponían los hoyos. Mediado un tiempo que no podemos precisar se dispuso el pavimento rojo, que selló los hoyos.

Desconocemos si los hogares se encendieron simultáneamente a las anteriores estructuras, como también, si no fue así, cuánto tiempo transcurrió entre la construcción de las citadas estructuras y los hogares. No obstante, la fecha sí data parte de las actividades que se llevaron a cabo en torno a los menhires, y pone de manifiesto que a comienzos del IV milenio el simbolismo que encarnaban estaba plenamente vigente, tratándose de un hito territorial que se relaciona con la posesión, control y derecho al uso de este territorio por parte de una comunidad productora que realiza aquí determinados rituales, como parecen evidenciar el suelo a base de hematites y los tres hogares, en los que los carbones se combinaron con cinabrio, hecho que prueba que su función no era de carácter cotidiano. La importancia de este sitio como hito territorial perduró más allá de la fecha obtenida, como se ha defendido en otras ocasiones (Gavilán y Vera 2005; Gavilán et al. 2020).

La datación que damos a conocer es la primera que se ha obtenido relacionada con el megalitismo en la provincia de Córdoba y, por ahora, la más antigua para el no funerario en Andalucía, aunque no feche el momento preciso de erección de estas arquitecturas, y, resulta ser, por el momento, de las más antiguas del megalitismo andaluz, sólo precedida por las del Dolmen de Alberite, donde tampoco se ha fechado la construcción (Ramos y Giles 1996), y similar a la de una de las fases de uso del Dolmen de El Palomar (Cabrero et al. 2005). En cuanto a los círculos, el onubense de la Pasada del Abad ha proporcionado unas fechas muy recientes, aunque se defiende una construcción entre el V-IV milenios (Linares 2009).
Para finalizar, no resulta posible plantear para el caso que nos ocupa la existencia de un comienzo del megalitismo asociado a poblados, como ocurre en el S de Portugal (Calado et al. 2004), ya que los restos materiales y las estructuras sólo aparecían en sectores muy concretos, como ya se ha indicado, y tampoco se localizaron restos en los terrenos inmediatos al túmulo que indiquen una ocupación espacial más amplia que permita defender la presencia de un poblado, salvo el de Sierra Palacios II, en la ladera de este conjunto calizo y a poco más de $1 \mathrm{~km}$ de distancia. Entre los restos materiales aportados por este poblado están presentes las cerámicas decoradas -aplicación de cordones, incisas, acanaladas y almagras-, pero son mayoría las no decoradas, con especial representación de las cazuelas carenadas. Para nosotros, Sierra Palacios II pertenece al Neolítico Final (Gavilán 20032004), pese a contar con una lezna de cobre, cuya presencia habrá que explicar en otro momento, pero, de entrada, debe tenerse en cuenta la proximidad de Sierra Palacios I, de la Edad del Cobre (Gavilán 2003-2004). Las fechas que se proponen para las ocupaciones correspondientes al Neolítico Final con cazuelas carenadas se sitúan en la primera y segunda mitad del IV milenio (Escacena y Rivero 2019), coincidiendo, en buena medida, con la datación que aquí damos a conocer.

La ausencia de las cazuelas carenadas entre los restos recuperados en torno a los menhires de Casas de Don Pedro puede deberse a dos factores: o bien la erección de los menhires es anterior a la aparición y extensión en esta zona de las citadas formas cerámicas, en cuyo caso, no puede establecerse una relación entre ellos y Sierra Palacios II; o bien responde al desarrollo de actividades completamente diferentes en ambos sitios, como parece lógico pensar ya que los menhires marcan un hito territorial, un espacio simbólico en el que las actividades desarrolladas poco o nada tiene en común con la esfera de la vida cotidiana, sino con la de las creencias y, en este caso, los menhires fueron erigidos por los habitantes de dicho poblado. De cualquier manera, es el único asentamiento que se conoce en la zona, hasta el momento, con suficiente entidad y, además, también debe tenerse en cuenta que al final del Neolítico los menhires pasan a formar parte de una estructura megalítica funeraria, que será objeto ya en el Calcolítico de remodelaciones, con la adición de un corredor de acceso. Sin duda, todo indica una continuidad en el poblamiento de la zona desde comienzos del IV milenio hasta el paso del III al II milenio. 
Para finalizar, sí queda patente que en los primeros años del IV milenio, por el momento, las sociedades productoras establecidas en la zona NO de la provincia de Córdoba comenzaron a erigir arquitecturas megalíticas, concretamente menhires, mientras que algunas cavidades aún tenían una función sepulcral, como tal vez puede deducirse de los restos localizados en Cueva Agustín, en Sierra Palacios (Gavilán 2003-2004), que concuerdan tipológicamente con los procedentes del exterior del Dolmen de las Casas de Don Pedro, es decir, en torno a los menhires. Para, a finales del Neolítico comenzar con los enterramientos en estructuras megalíticas ya funerarias.

\section{AGRADECIMIENTOS}

Este trabajo se ha realizado en el marco del Grupo de Investigación HUM-838: Poder y territorio desde la Prehistoria a la Edad Media, subvencionado por la Consejería de Educación y Ciencia de la Junta de Andalucía.

\section{BIBLIOGRAFÍA}

BARRIOS, J.; GAVILÁN, B.; MARTÍN, J. C.; MONTEALEGRE, L. (2014): Territorio y materias primas: el dolmen de las Casas de Don Pedro (Belmez, Córdoba), II Congreso de Prehistoria de Andalucía. Movilidad, contacto y cambio, Sevilla, 391-398.

CABRERO GARCÍA, R.; AMBROSIANI FERNÁNDEZ, J.; GUIJO MAURI, J. M.; GÓMEZ MURGA, E. (2005): Estudio de restos humanos procedentes del Dolmen de Cañada Real depositados en el Departamento de Anatomía y Embriología Humanas de la Facultad de Medicina de la Universidad de Sevilla, Spal 14, 59-74.

DOI: https://doi.org/10.12795/spal.2005.i14.02

CALADO MENDES, D.; NOCETE CALVO, F.; CÁMALICH MASSIEU, M. D.; MARTÍN SOCAS, D.; NIETO LIÑÁN, J. M.; ALEX TURQ, E.; RODRÍGUEZ BAYONA, M. (2004): Los poblados con menhires del Algarve Occidental: Nuevas perspectivas para la explicación de las primeras sociedades sedentarias en el Suroeste Peninsular, Jornadas Temáticas Andaluzas de Arqueología, 195-204.

ESCACENA CARRASCO, J. L.; GARCÍA RIVERO, D. (2019): Producción neolítica de sal marina en La Marismilla (La Puebla del Río, Sevilla). Datos renovados e hipótesis complementarias, LVCENTVM XXXVIII, 9-26 DOI: https://doi.org/10.14198/LVCENTVM2019.38.01
GAVILÁN, B. (1987): Excavación arqueológica de urgencia en el Dolmen de las Casas de Don Pedro (Belmez, Córdoba). Anuario Arqueológico de Andalucía, 1986. III: Actividades de Urgencia, Junta de Andalucía, Sevilla, 118-120.

GAVILÁN, B. (2003-2004): El Alto Valle del Guadiato durante la Prehistoria Reciente: El poblamiento Neolítico y Calcolítico, Espacio, Tiempo y Forma. Serie I: Prehistoria y Arqueología 16-17, 119-170. DOI: https://doi.org/10.5944/etfi.16-17.2003.4753

GAVILÁN, B.; MAS, M., RODRÍGUEZ, J.; SOLÍS, M.; GARRIDO, E. (2020): Hallazgos de cinabrio en contextos del Neolítico cordobés: la Cueva de los Murciélagos (Zuheros) y el dolmen de Casas de Don Pedro (Belmez), El oro rojo en la Antigüedad. Perspectivas de investigación sobre los usos y aplicaciones del cinabrio entre la Prehistoria y el fin del mundo antiguo (M. Zarzalejos Prieto, P. Hevia Gómez, L. Mansilla Plaza, eds.), Madrid, 161-186.

GAVILÁN, B.; VERA, J. C. (2005): Neolítico y megalitismo prefunerario en Andalucía, III Congreso del Neolítico en la Península Ibérica (R. Ontañón, C., García-Moncó, P. Arias, eds.), Santander, 535-541.

LESTÓN GÓMEZ, M. (2011): Las excavaciones y los descubrimientos, El Dolmen de Dombate. Arqueología, Arquitectura y Conservación (F. Cebrián del Moral, J. Yáñez, M. Lestón, F. Vidal, F. Carrera, eds.), A Coruña, 139-227.

LINARES CATELA, J. A. (2009): El círculo megalítico de la Pasada del Abad (Rosal de la Frontera, Huelva). El megalitismo no funerario de la Rivera del Chanza, IV Encuentro de Arqueología del Suroeste Peninsular, Huelva, 174-208.

RAMOS MUÑOZ, J.; GILES PACHECO, F. (eds.) (1996): El Dolmen de Alberite (Villamartín). Aportaciones a las Formas Económicas y Sociales de las Comunidades Neolíticas en el Noroeste de Cádiz, Cádiz. 